\title{
Congenital Fusion of the Mandible and Maxilla- A Case Report from Ajman, United Arab Emirates
}

\author{
Mohamed Said Hamed ${ }^{1, *}$, Prathibha Prasad ${ }^{2}$ \\ ${ }^{1}$ Professor of Oral and Maxillofacial Surgery, College of Dentistry, Gulf medical University, Ajman, United Arab Emirates \\ ${ }^{2}$ Lecturer, Specialist in Oral Pathology, College of Dentistry, Gulf medical University, Ajman, United Arab Emirates \\ Corresponding author: mohamedsaid771@yahoo.com
}

Received October 12, 2014; Revised November 23, 2014; Accepted December 21, 2014

\begin{abstract}
Congenital fusion of the mandible to maxilla is a rare phenomenon. The fusion can be a bony fusion in which case it's referred to as Syngnathia or soft tissue fusion when it's addressed as Synechiae. [1] Soft tissues fusion is involved most often in the oral cavity but it may involve both hard and soft tissues of the child. This results in inability of child to thrive as it doesn't allow mouth opening and feeding. This is a case report of a 3-day-old baby boy with partial maxilla-mandibular fusion from right canine to posterior jaw who was brought to Oral and Maxillofacial department of the dental centre in GMC Hospital, Ajman, UAE for treatment because of inability to thrive.
\end{abstract}

\section{Keywords: congenital fusion, syngnathia}

Cite This Article: Mohamed Said Hamed, and Prathibha Prasad, "Congenital Fusion of the Mandible and Maxilla- A Case Report from Ajman, United Arab Emirates." International Journal of Dental Sciences and Research, vol. 2, no. 6C (2014): 8-9. doi: 10.12691/ijdsr-2-6C-2.

\section{Introduction}

Congenital fusion of the mandible to maxilla is a rare phenomenon. The fusion can be a bony fusion in which case it's referred to as Syngnathia or soft tissue fusion when it's addressed as Synechiae. [1] Soft tissues fusion is involved most often in the oral cavity but it may involve both hard and soft tissues of the child. This results in inability of child to thrive as it doesn't allow mouth opening and feeding. Treatment should be provided as early as possible as it could eventually result in the ankylosis of the temporomandibular joint. [2] The bony fusion can be further classified as partial or complete and then as syndromic and nonsyndromic. The etiology remains unknown although various theories have been postulated. Treatment has to be case to case basis [1].

\section{Case Report}

A 3-day-old baby boy with partial maxilla-mandibular fusion from right canine to posterior jaw was brought to Oral and Maxillofacial department of the dental centre in GMC Hospital, Ajman for treatment because of inability to thrive. The patient's jaws were found to be fused in the posterior regions on the right side. In the anterior region and left side of the jaw there was no fusion. Except for the fact that the parents were consanguineous, the medical history of both of them was insignificant. The mother gave a history of healthy pregnancy and denied any illness, trauma, or drug use. The baby was their 1st child born by normal vaginal delivery. Both temporomandibular joints seem to function normally.

\section{Discussion}

Syngnathia is a quite a rare disorder and is often seen with syngnathia. [3] This pathology is associated with various syndromes such as Van der Woude, cleft palate alveolar syngnathia, and oromandibular limb hypogenesis syndrome. Since the first case reported in 1936 by Burket, only 27 cases of syngnathia have been reported. [4] Laster et al [5] reported 24 cases. Daniels6reported a case of bony fusion of the mandible to the maxilla and to the zygomatic complex. Parkins and Boamah [7] reported a case of fusion of the jaws, synechiae of the buccal mucosa and the gingiva, with cleft of the hard palate.

Gartlan et al. (1993) [8] reviewed synechiaeand were relegated as buccopharyngeal membrane remnants or as ectopic membranes on the substratum of their surmised inchoation. Particularly its isolated occurrence, bony fusion (syngnathia), is profoundly infrequent. A very few cases reported in the literature are in description, inconsistently erratic and perplexing in nomenclature and with circumscribed subsidiary conventional imaging [8].

The etiology is unclear. Neural crest cell derived osteogenic patterning is affected in the absence of Foxc1, as osteoblasts develop ectopically in the maxillary prominence and fuse with the dentary bone. Kimberley Inman has provided evidence that Foxc1 and Fgf8 genetically interact and it is Fgf8 dosage responsible for variation in the syngnathic phenotype [9]. 
Because the neonate is not able to open the mouth or feed normally, Maxillomandibular fusion is usually discovered immediately after birth [10].

The relegation for syngnathia regarding other anomalies was proposed by Dawson et al [11] as follows: type 1: simple syngnathia, with no other anomalies in the head and neck; type 2: intricate syngnathia, with subgroups, type 2a: syngnathia coexistent with aglossia and type $2 \mathrm{~b}$ : syngnathia coexistent with agenesis or hypoplasia of proximal mandible.

After their experience with a case of fusion of the mandible to the zygomatic involute and maxillary tuberosity, however, Laster et al [5] proposed a modified relegation for bony syngnathia: type 1a: simple anterior syngnathia, characterized without other congenital deformity in the head and neckand by bony fusion of the alveolar ridge only and; type 1b: complex anterior syngnathia, characterized by associated with other congenital deformity in the head and neckand by bony fusion of the alveolar ridges only and; type 2a: simple zygomaticomandibularsyngnathia characterized by bony fusion of the mandible to the zygomatic complex causing only mandibular micrognathia; type 2b: complex zygomaticomandibularsyngnathia, characterized by bony fusion of the mandible to the zygomatic complex and associated with clefts or temporomandibular joint ankylosis.

The rigor of this disorder has no fine-tuned pattern. The range of jaw fusion elongates from consummate fusion, unilateral fusion, or bilateral fusion with a diminutive anterior opening. [10] Our case belonged to the unilateral fusion type.

Interfered with feeding, breathing, general health of the patient, this condition is problematic and (aspiration pneumonitis), growth and development, induction of anesthesia (intubation) etc. The infrequency of this condition imposes some inhibitions on standardization of the treatment [8].

It is more problematic in involute cases in terms of jaw function and its outcome. Dawson, et al [11] proposed a system of relegation and elaborated on treatment recommendations focusing on functional outcome. In the management of any newborn with trismus, the airway is the first priority to be secured. Afterwards, feeding problems should be overcome by placing a nasogastric or gastronomy tubes.

There is high rate of association between bony syngnathia and systemic malformations and the patient should be under the supervision of a team of clinicians to aid proper diagnosis and treatment [12].

Under general anesthesia (blind intubation or via tracheostomy), surgical division of the bony fusion, is the optimal treatment for the simple syngnathia in isolated occurrence or cases with the availability of other anatomic abnormalities. The infant should be encouraged to feed normally as soon as possible [8] and proper physical therapy should be started immediately,

\section{References}

[1] Dean J. Trigg, Ifan T. Mau, Kristina W. Rosbe. Complete Bony Syngnathia Report of a Case and Review. Arch Otolaryngol Head Neck Surg. 2007; 133 (2): 187-190.

[2] Seyyed Hossein Mortazavi, Mohammad Hosein Kalantar Motamedi. Congenital Fusion of the Jaws. Indian J Pediatr 2007; 74 (4): 416-8.

[3] Shams M G, Motamedi M HK, Abad H LD. Congenital fusion of the maxilla and mandible: brief case report. Oral Surg Oral Med Oral Pathol Oral Radiol Endod. 2006; 102: e1-e3.

[4] Burket L N. Congenital bony ankylosis and facial hemiatrophy. J Am Med Assoc. 1936; 106: 1719-1722.

[5] Laster Z, Temkin D, Zarfin Y, Kushnir A. Complete bony fusion of the mandible to the zygomatic complex and maxillary tuberosity: case report and review. Int J Oral Maxillofac Surg. 2001; 30: 75-79.

[6] Daniels J SM. Congenital maxillomandibular fusion: a case report and review of the literature. J Craniomaxillofac Surg. 2004; 32: 135-139.

[7] Parkins G E, Boamah M O. Congenital maxillomandibular syngnathia: case report. J Craniomaxillofac Surg. 2009; 37: 276278.

[8] Ismail Yazdi, Amir Hossein Fakhraee. Congenital Fusion of Maxilla and Mandible (Bony Syngnathia): A Case Report. Archives of Iranian Medicine. 2005; 203 (20): 1-4.

[9] Kimberly E. Inman, Patricia Purcell, Tsutomu Kume, Paul A. Trainor. Interaction between Foxc1 and Fgf8 during Mammalian Jaw Patterning and in the Pathogenesis of Syngnathia. PLoS Genetics, 2013; 9 (12): e100394.

[10] Lorena Pingarrón Martín, Mercedes Martín Pérez, Elena Gómez García, Javier González Martín-Moro, Jose Ignacio Rodríguez González, Miguel Burgueño García. Atypical Case of Congenital Maxillomandibular Fusion with Duplication of the Craniofacial Midline. Craniomaxillofac Trauma Reconstr. Jun 2011; 4 (2): 113120.

[11] Dawson K H, Gruss J S, Myall R W. Congenital bony syngnathia: a proposed classification. Cleft Palate Craniofac J. 1997; 34: 141146.

[12] M Mir, S Iqbal, A Hafeez, H Zargar, A Rasool, M Mohsin, A Darzi. Syngnathia without Any Other Associated Anomaly: A Very Rare Case Report. The Internet Journal of Plastic Surgery. 2006; 4 (1). 